\author{
B. Usna Banu and G. P. Youvaraj
}

\title{
RADIUS OF CONVEXITY OF SECTIONS OF A CLASS OF CLOSE-TO-CONVEX FUNCTIONS OF ORDER $\alpha$
}

\begin{abstract}
In this paper we study radius of convexity of sections of a class of univalent close-to-convex functions on $\mathbb{D}=\{z \in \mathbb{C}$ : $|z|<1\}$. For functions in this class, coefficient bounds, an integral representation and radius of convexity of $n^{\text {th }}$ sections have been obtained.
\end{abstract}

KEY WORDS: univalent, close-to-convex, starlike and convex functions.

AMS Mathematics Subject Classification: 30C45.

\section{Introduction}

Let $\mathcal{A}$ be the set of all analytic functions of the form $f(z)=z+\sum_{k=2}^{\infty} a_{k} z^{k}$ in the unit disc $\mathbb{D}$ and let $\mathcal{S}$ denote the class of all univalent (one-to-one and analytic) functions $f \in \mathcal{A}$. Let $\mathcal{C}, \mathcal{S}^{*}$ and $\mathcal{K}$ denote the subclasses of $\mathcal{S}$ that are convex, starlike with respect to origin and close-to-convex functions respectively. It is well known that $\mathcal{C} \subset \mathcal{S}^{*} \subset \mathcal{K} \subset \mathcal{S}$. Let $S_{n}(f)=z+\sum_{k=2}^{n} a_{k} z^{k}$ be the $n^{\text {th }}$ section of $f$.

In [8], Szego proved that every section $S_{n}(f)$ of $f \in \mathcal{S}$ is univalent in the disk $|z|<\frac{1}{4}$ and $\frac{1}{4}$ is the best possible as $S_{2}(k(z))=z+2 z^{2}$ is univalent in $|z|<\frac{1}{4}$, where $k(z)=\frac{z}{(1-z)^{2}}$ is Koebe function, extremal function of the class $\mathcal{S}$.

Various problems about sections have been solved for subclasses $\mathcal{C}, \mathcal{S}^{*}, \mathcal{K}$ in [1], [3], [4], [6]. In [2], MacGregor considered the class $\mathcal{R}=\{f \in \mathcal{A}$ : $\left.\operatorname{Re}\left(f^{\prime}(z)\right)>0, z \in \mathbb{D}\right\}$ and proved that every section $S_{n}(f)$ of $f \in \mathcal{R}$ is univalent in $|z|<\frac{1}{2}$ and $\frac{1}{2}$ is the best constant. In [5], Ponnusamy et.al. considered the class $\mathcal{F}=\left\{f \in \mathcal{A} / \operatorname{Re}\left(1+\frac{z f^{\prime \prime}(z)}{f^{\prime}(z)}\right)>-\frac{1}{2}, z \in \mathbb{D} \backslash\{0\}\right\}$ 
and proved that every section of a function $f \in \mathcal{F}$ is convex in the disc $|z|<\frac{1}{6}$. The radius $\frac{1}{6}$ cannot be replaced by a larger one. In this paper, we consider the class

$$
\mathcal{F}_{\alpha}=\left\{f \in \mathcal{A} / \operatorname{Re}\left(1+\frac{z f^{\prime \prime}(z)}{f^{\prime}(z)}\right)>-\frac{\alpha}{2}, 0 \leq \alpha \leq 1, z \in \mathbb{D} \backslash\{0\}\right\}
$$

and find coefficient bound, distortion theorem and radius of convexity of sections of functions in this class.

In Theorem 1, though the result in $(a)$ below had been proved in [7] for a wider range, we prove it here for the sake of completeness of this paper.

\section{Main theorems}

\section{Coefficient bound and distortion theorem} have

Theorem 1. If $f(z)=z+\sum_{n=2}^{\infty} a_{n} z^{n}$ belongs to $\mathcal{F}_{\alpha}, 0 \leq \alpha \leq 1$, then we

(a) $\left|a_{n}\right| \leq \frac{\Gamma(n+1+\alpha)}{n ! \Gamma(2+\alpha)}$ for $n \geq 2$, equality holds for the extremal function of the class $\mathcal{F}_{\alpha}$, which is given by $f_{\alpha}=\frac{1}{1+\alpha}\left(\frac{1}{(1-z)^{1+\alpha}}-1\right)$;

(b) $\left|\frac{z f^{\prime \prime}(z)}{f^{\prime}(z)}\right|<\frac{(2+\alpha) r}{1-r}$ for $|z|=r ;$

(c) $\frac{1}{(1+r)^{2+\alpha}}<\left|f^{\prime}(z)\right|<\frac{1}{(1-r)^{2+\alpha}}$.

Proof. As $f \in \mathcal{F}_{\alpha}$, we can write $1+\frac{z f^{\prime \prime}(z)}{f^{\prime}(z)}+\frac{\alpha}{2}=\left(1+\frac{\alpha}{2}\right) P(z)$, where $P(z)=1+\sum_{n=1}^{\infty} p_{n} z^{n}$ is such that $\operatorname{Re}(P(z))>0$ having $\left|p_{n}\right| \leq 2, n \geq 1$. Also,

$$
\begin{aligned}
\frac{2+\alpha}{2}+\frac{z f^{\prime \prime}(z)}{f^{\prime}(z)} & =\frac{2+\alpha}{2}(P(z)) \\
\frac{\sum_{2}^{\infty} n(n-1) a_{n} z^{n-1}}{1+\sum_{2}^{\infty} n a_{n} z^{n-1}} & =\frac{2+\alpha}{2}\left(\sum_{n=1}^{\infty} p_{n} z^{n}\right) \\
\sum_{1}^{\infty} n(n+1) a_{n+1} z^{n} & =\frac{2+\alpha}{2}\left(1+\sum_{1}^{\infty}(n+1) a_{n+1} z^{n}\right)\left(\sum_{n=1}^{\infty} p_{n} z^{n}\right) .
\end{aligned}
$$

Equating coefficient of $z$ we have as $\left|p_{1}\right|<2$,

$$
1.2\left|a_{2}\right|=\frac{2+\alpha}{2}\left|p_{1}\right|<(2+\alpha) ; \text { (i.e) }\left|a_{2}\right|<\frac{2+\alpha}{2} .
$$


For $n \geq 2$ we have

$$
\begin{aligned}
n(n+1)\left|a_{n+1}\right| & \leq \frac{2+\alpha}{2}\left(\left|p_{n}\right|+\sum_{k=1}^{n-1}(k+1)\left|a_{k+1}\right|\left|p_{n-k}\right|\right) \\
& <(2+\alpha) \sum_{k=1}^{n} k\left|a_{k}\right| .
\end{aligned}
$$

We claim that for $n \geq 2$,

$$
n(n+1)\left|a_{n+1}\right| \leq(2+\alpha) \sum_{k=1}^{n} k\left|a_{k}\right| \leq \frac{(2+\alpha)(3+\alpha) \ldots(n+1+\alpha)}{(n-1) !} .
$$

For $n=2$,

$$
\begin{aligned}
2.3\left|a_{3}\right| & \leq(2+\alpha) \sum_{k=1}^{2} k\left|a_{k}\right|<(2+\alpha)(3+\alpha) \\
\left|a_{3}\right| & \leq \frac{(2+\alpha)(3+\alpha)}{3 !}
\end{aligned}
$$

Now assume that for $n=m$, the following is true.

$$
m(m+1)\left|a_{m+1}\right| \leq(2+\alpha) \sum_{k=1}^{m} k\left|a_{k}\right| \leq \frac{(2+\alpha)(3+\alpha) \ldots(m+1+\alpha)}{(m-1) !} .
$$

Next we consider $n=m+1$,

$$
\begin{aligned}
(m+1)(m+2)\left|a_{m+2}\right| & \leq(2+\alpha) \sum_{k=1}^{m+1} k\left|a_{k}\right| \\
& =(2+\alpha) \sum_{k=1}^{m} k\left|a_{k}\right|+(2+\alpha)(m+1)\left|a_{m+1}\right| \\
& =\frac{(2+\alpha)(3+\alpha) \ldots(m+2+\alpha)}{m !}
\end{aligned}
$$

Therefore, $\left|a_{m+2}\right| \leq \frac{(2+\alpha)(3+\alpha) \ldots(m+2+\alpha)}{(m+2) !}$. This bound is sharp as $f_{\alpha}=\frac{1}{1+\alpha}\left(\frac{1}{(1-z)^{1+\alpha}}-1\right) \in \mathcal{F}_{\alpha}$. To Prove $(b)$, we know by definition of $\mathcal{F}_{\alpha}, 1+\frac{z f^{\prime \prime}(z)}{f^{\prime}(z)} \prec \frac{1+(1+\alpha) z}{1-z}$ for $f \in \mathcal{F}_{\alpha}$. Therefore, $\frac{z f^{\prime \prime}(z)}{f^{\prime}(z)} \prec \frac{(2+\alpha) z}{1-z}=$ $h_{\alpha}(z)$, where $\prec$ denotes subordination. Hence $\left|\frac{z f^{\prime \prime}(z)}{f^{\prime}(z)}\right|<\frac{(2+\alpha) r}{1-r}$. From 
the above, it is clear that $f^{\prime}(z) \prec e^{\int^{z} \frac{h_{\alpha}(t)}{t} d t}=\frac{1}{(1-z)^{2+\alpha}}$ and hence $(c)$ holds.

Theorem 2. For $f \in \mathcal{F}_{\alpha}$, if $f(z)=s_{n}(z)+\sigma_{n}(z)$, where $s_{n}(z)=$ $z+\sum_{k=2}^{n} a_{k} z^{k}$ and $\sigma_{n}(z)=\sum_{k=n+1}^{\infty} a_{k} z^{k}$, then for $|z|=r$ we have

$$
\begin{aligned}
\left|\sigma_{n}^{\prime}(z)\right|<\frac{\Gamma(n+3+\alpha)}{\Gamma(2+\alpha)(n+1) !} \frac{r^{n+1}}{(1-r)^{n+3+\alpha}}, \\
\left|z \sigma_{n}^{\prime \prime}(z)\right|<\frac{\Gamma(n+4+\alpha)}{\Gamma(2+\alpha)(n+1) !} \frac{r^{n+2}}{(1-r)^{n+4+\alpha}} .
\end{aligned}
$$

Proof. Consider $\left|\sigma_{n}(z)\right| \leq \sum_{n+1}^{\infty}\left|a_{k}\right||z|^{k}<\sum_{n+1}^{\infty} \frac{\Gamma(k+1+\alpha)}{\Gamma(2+\alpha) k !} r^{k}=\sigma_{n 0}(r)$, where $\sigma_{n 0}(r)$ is the remainder, after $n$ terms, of the extremal function

$$
f_{0}(r)=\frac{1}{1+\alpha}\left(\frac{1}{(1-r)^{1+\alpha}}-1\right) .
$$

By Integral form of remainder of a Taylor series, we get

$$
\begin{aligned}
\left|\sigma_{n 0}(r)\right| & =\left|\int_{0}^{r} \frac{f_{0}^{(n+1)}(t)(r-t)^{n}}{n !} d t\right| \\
& <\int_{0}^{r}\left|\frac{\Gamma(n+2+\alpha)}{\Gamma(2+\alpha) n !} \frac{(r-t)^{n}}{(1-t)^{n+2+\alpha}}\right| d t \\
& <\frac{\Gamma(n+2+\alpha)}{\Gamma(2+\alpha) n !(1-r)^{n+2+\alpha}} \int_{0}^{r}(r-t)^{n} d t \quad \text { as } \quad 0<t<r \\
& =\frac{\Gamma(n+2+\alpha)}{\Gamma(2+\alpha)(n+1) !} \frac{r^{n+1}}{(1-r)^{n+2+\alpha}} .
\end{aligned}
$$

Hence

$$
\left|\sigma_{n}(z)\right| \leq \frac{\Gamma(n+2+\alpha)}{\Gamma(2+\alpha)(n+1) !} \frac{r^{n+1}}{(1-r)^{n+2+\alpha}} \quad \forall z \in \mathbb{D} .
$$

Similarly for all $z \in \mathbb{D}$ we can obtain,

$$
\begin{aligned}
\left|\sigma_{n}^{\prime}(z)\right| & \leq \frac{\Gamma(n+3+\alpha)}{\Gamma(2+\alpha)(n+1) !} \frac{r^{n+1}}{(1-r)^{n+3+\alpha}} \\
\left|z \sigma_{n}^{\prime \prime}(z)\right| & \leq \frac{\Gamma(n+4+\alpha)}{\Gamma(2+\alpha)(n+1) !} \frac{r^{n+2}}{(1-r)^{n+4+\alpha}}
\end{aligned}
$$


Theorem 3 (Integral representation of the class). If $f \in \mathcal{F}_{\alpha}$, then the integral representation of $f(z)$ is of the form

$$
f^{\prime}(z)=\frac{e^{[(2+\alpha) / 2] \int_{0}^{z}[P(t) / t] d t}}{z^{(2+\alpha) / 2}}
$$

for some $P(z)$ with $\operatorname{Re}(P(z))>0$ and vice versa.

Proof. As $f \in \mathcal{F}_{\alpha}$, we have $1+\frac{z f^{\prime \prime}(z)}{f^{\prime}(z)}+\frac{\alpha}{2}=\left(1+\frac{\alpha}{2}\right) P(z)$, where $P(z)$ is such that $\operatorname{Re}(P(z))>0$. Therefore, we have

$$
\text { (i.e) } \begin{aligned}
1+\frac{z f^{\prime \prime}(z)}{f^{\prime}(z)} & =\left(1+\frac{\alpha}{2}\right) P(z)-\frac{\alpha}{2} ; \\
\frac{d}{d z} \log \left(z f^{\prime}(z)\right) & =\frac{2+\alpha}{2} P(z)-\frac{\alpha}{2} \\
\frac{d}{d z} \log \left(z f^{\prime}(z)\right) & =\frac{2+\alpha}{2} \frac{P(z)}{z}-\frac{\alpha}{2 z} .
\end{aligned}
$$

Integrating we get,

$$
\begin{aligned}
\log \left(z f^{\prime}(z)\right) & =\frac{2+\alpha}{2} \int_{0}^{z} \frac{P(t)}{t} d t-\int_{0}^{z} \frac{\alpha d t}{2 t} \\
& =\frac{2+\alpha}{2} \int_{0}^{z} \frac{P(t)}{t} d t-\frac{\alpha}{2} \log (z) .
\end{aligned}
$$

Taking exponentiation we obtain,

$$
\begin{aligned}
z f^{\prime}(z) & =z^{-\alpha / 2} e^{\frac{2+\alpha}{2} \int_{0}^{z} \frac{P(t)}{t} d t}, \\
f^{\prime}(z) & =\frac{e^{[(2+\alpha) / 2] \int_{0}^{z}[P(t) / t] d t}}{z^{(2+\alpha) / 2}} .
\end{aligned}
$$

Hence the proof and converse can be proved by retracing the above steps.

Theorem 4. For $f \in \mathcal{F}_{\alpha}$, if $f(z)=s_{n}(z)+\sigma_{n}(z)$, where $s_{n}(z)=$ $z+\sum_{k=2}^{n} a_{k} z^{k}$ and $\sigma_{n}(z)=\sum_{k=n+1}^{\infty} a_{k} z^{k}$, then every section $s_{n}(z)$ of functions in this class is convex in the disk $|z|<\frac{1}{2(2+\alpha)}$ and this radius cannot be replaced by larger one. 
Proof. Consider Re $\left(1+\frac{z s_{2}^{\prime \prime}(z)}{s_{2}^{\prime}(z)}\right)=1+\frac{2 a_{2} z}{1+2 a_{2} z}$. Then by Theorem 1 , we have $\left|a_{2}\right|<\frac{2+\alpha}{2}$ and hence $\operatorname{Re}\left(1+\frac{z s_{2}^{\prime \prime}(z)}{s_{2}^{\prime}(z)}\right)>1-\frac{2\left|a_{2}\right||z|}{1-2\left|a_{2}\right||z|} \geq 1-$ $\frac{(2+\alpha)|z|}{1-(2+\alpha)|z|} ; \operatorname{Re}\left(1+\frac{z s_{2}^{\prime \prime}(z)}{s_{2}^{\prime}(z)}\right)>\frac{1-2(2+\alpha)|z|}{1-(2+\alpha)|z|}=0$ at $|z|=\frac{1}{2(2+\alpha)}$. By Maximum Principle for harmonic functions, we have $\operatorname{Re}\left(1+\frac{z s_{2}^{\prime \prime}(z)}{s_{2}^{\prime}(z)}\right)>0$ for $|z| \leq \frac{1}{2(2+\alpha)}$. Now for $n>2$, we obtain

$$
\operatorname{Re}\left(1+\frac{z s_{n}^{\prime \prime}(z)}{s_{n}^{\prime}(z)}\right)>1-\left|\frac{z f^{\prime \prime}(z)}{f^{\prime}(z)}\right|-\frac{\left|\frac{z f^{\prime \prime}(z)}{f^{\prime}(z)}\right|\left|\sigma_{n}^{\prime}(z)\right|+\left|z \sigma_{n}^{\prime \prime}(z)\right|}{\left|f^{\prime}(z)\right|-\left|\sigma_{n}^{\prime}(z)\right|}
$$

Then by Theorem 2, we have

$$
\begin{aligned}
& >1-\frac{(2+\alpha) r}{1-r} \\
& -\frac{\frac{r^{n+2} \Gamma(n+3+\alpha)}{(1-r)^{n+4+\alpha} \Gamma(2+\alpha)(n+1) !}[2+\alpha+n+3+\alpha]}{\left(\frac{\Gamma(2+\alpha)(n+1) !(1-r)^{n+3+\alpha}-(1+r)^{2+\alpha} \Gamma(n+3+\alpha) r^{n+1}}{(1+r)^{2+\alpha} \Gamma(2+\alpha)(n+1) !(1-r)^{n+3+\alpha}}\right)} \\
& \operatorname{Re}\left(1+\frac{z s_{n}^{\prime \prime}(z)}{s_{n}^{\prime}(z)}\right)>\frac{1-(3+\alpha) r}{1-r} \\
& -\frac{r^{n+2} A_{0}(1+r)^{2+\alpha}[n+5+2 \alpha]}{(1-r)\left((1-r)^{n+3+\alpha}-A_{0}(1+r)^{2+\alpha} r^{n+1}\right)}
\end{aligned}
$$

where $A_{0}=\frac{\Gamma(n+3+\alpha)}{\Gamma(2+\alpha)(n+1) !}$. Then $\operatorname{Re}\left(1+\frac{z s_{n}^{\prime \prime}(z)}{s_{n}^{\prime}(z)}\right)>0$ provided

$$
\frac{1-(3+\alpha) r}{1-r}>\frac{r^{n+2} A_{0}(1+r)^{2+\alpha}[n+5+2 \alpha]}{(1-r)\left((1-r)^{n+3+\alpha}-(1+r)^{2+\alpha} A_{0} r^{n+1}\right)} .
$$

Therefore, we have to prove

$$
\begin{aligned}
r^{n+2} A_{0}(1+r)^{2+\alpha}[n+5+2 \alpha]< & {[1-(3+\alpha) r]\left((1-r)^{n+3+\alpha}\right.} \\
& \left.-(1+r)^{2+\alpha} A_{0} r^{n+1}\right) \\
r^{n+1} A_{0}(1+r)^{2+\alpha}[1+(n+2+\alpha) r]< & {[1-(3+\alpha) r](1-r)^{n+3+\alpha} . }
\end{aligned}
$$

On $|z|=\frac{1}{2(2+\alpha)}$, above inequality becomes

$$
A_{0}(5+2 \alpha)^{2+\alpha}[n+6+3 \alpha]<(1+\alpha)(3+2 \alpha)^{n+3+\alpha}
$$


which is true for all $n \geq 3$ and $0 \leq \alpha \leq 1$. By Maximum Principle for harmonic functions, we have $\operatorname{Re}\left(1+\frac{z s_{n}^{\prime \prime}(z)}{s_{n}^{\prime}(z)}\right)>0$ in the disk $|z| \leq \frac{1}{2(2+\alpha)}$. Hence the radius of convexity of sections of $f \in \mathcal{F}_{\alpha}$ is $|z|=\frac{1}{2(2+\alpha)}$.

\title{
References
}

[1] Duren P.L., Univalent Functions, Springer Verlog, 1983.

[2] MacGregor T.H., Functions whose derivatives has a positive real part, Trans. Amer. Math. Soc., 104(1962), 532-537.

[3] Robertson M.S., The partial sums of multivalently starlike functions, Ann. of Math., 42(1941), 829-838.

[4] Ruschweye St., On the radius of univalence of partial sums of convex functions, Bull. Lond. Math. Soc., 4(1972), 367-369.

[5] Ponnusamy S., Sahoo S.K., Yanagihara H., Radius of convexity of partial sums of functions in the close to convex family, Nonlinear Analysis, 95(2014), 219-228.

[6] Small T.S., A note on the partial sums of convex schlicht functions, Bull. Lond. Math. Soc., 2(1970), 165-168.

[7] Suffridge T., Some special classes of conformal mappings, In Handbook of complex analysis: geometric function theory (Edited by $\mathrm{K}^{*}$ uhnau), Elsevier, Amsterdam, 2(2005), 309-338.

[8] Szego G., Zur Theorie der Schlichten Abbildungen, Math. Ann., 100(1) (1928), 188-211.

\author{
B. UsNa BANU \\ Ramanujan Institute for Advanced Study in Mathematics \\ UNIVERSITY OF MADRAS \\ Chepauk, Chennai 600 005, India \\ e-mail: usnabanu1005@gmail.com \\ G. P. YouvaraJ \\ Ramanujan Institute for Advanced Study in Mathematics \\ UNIVERSITY OF MADRAS \\ Chepauk, Chennai 600 005, India \\ e-mail: youvarajgp@yahoo.com
}

Received on 14.10.2017 and, in revised form, on 10.05.2018. 